\title{
ENGORDE DE OVINOS PELIBUEY CON ZERANOL (Ralgro) EN EXPLOTACION SE MIINTENSIVA EN PUCALLPA
}

Julio Rosales

Javier Pomiano**

\section{RESUMEN}

La alimentación de ovinos se basa principalmente en pastos naturales. Sin embargo, existe posibilidades de suplementación y el uso de aditivos no nutricionales para mejorar la producción animal. El presente estudio se realizó en el Centro Regional de Investigación del Instituto de investigaciones de la A mazonía Peruana, Pucallpa. El objetivo fue determinar el efecto biológico y económico del zeranol en el engorde de ovinos Pelibuey bajo crianza-intensiva. Se trabajó con 16 ovinos con cuatro tratamientos T1 (Pasto solo), T2 (Pasto + Alimento Balanceado), T3 (Pasto + Alimento Balanceado + Zeranol) y T4 (Pasto + Zeranol), con una duración experimental de 63 días. Se evaluó la ganancia de peso, rendimiento de carcasa, y beneficio económico. La mayor ganancia de peso $(\mathrm{P}<0.05)$ se obtuvo con el tratamiento T3 con 181 g/animal/día, seguido por los tratamiento T2, T4 y T1 con 149; 148; y 121 $\mathrm{g} / \mathrm{animal} / \mathrm{dí}_{\mathrm{a}}$ respectivamente. Para el rendimiento de carcasa se encontró superioridad signiticativa $(P<0.05)$ entre el tratamiento T3 con $50.47 \%$, seguido por los tratamientos T2, T4 y T1 con $48.25 \%$; $48.21 \%$ y $45.75 \%$, respectivamente. Económicamente, el tratamiento T3 reportó mejor beneficio neto por $\mathrm{kg}$ de carne. Se concluye que el uso del anabólico zeranol origina efectos biológicos y económicos positivos en ovinos de pelo Pelibuey.

\section{INTRODUCCION}

En la Amazonía peruana la alimentación de ovinos de pelo se basa principalmente en pastos naturales, conformado por gramíneas de pobre producción y baja calidad nutritiva. Esto determina una baja producción animal. Además de ésta existen otros factores, tales como el mal manejo y las condiciones deficientes de sanidad que limitan la expresión de la verdadera capacidad genética en cuanto a productividad. Sin embargo, existen posibilidades

\footnotetext{
* Investigador Pecuario del Centro Regional de Investigación del Instituto de Investigaciones de la A mazonía Peruana, Filial U cayali.

** Tesista de la U niversidad N acional A graria de la Selva.
} 
de un suplemento con raciones balanceadas, y el uso de aditivos no nutricionales que actúan como anabolizantes, coadyuvando al crecimiento y consecuentemente una mayor ganancia de peso.

El objetivo de este estudio es determinar el efecto del zeranol en el engorde de ovinos Pelibuey bajo el sistema de crianza semiintensivo.

\section{REVISION DE LITERATURA}

Durante 1960 los científicos de la International Mineral Chemical Corporation, (1982), descubrieron el zeranol o zearalanol que es una lactosa del ácido U-6 (6, 10 Dihidroxiundecil) Resorcílico, que viene a ser el principio del Ralgro. Este es considerado como un compuesto químico no hormonal (Vara y Moreno, 1988). La acción del zeranol empieza después del implante, con el aumento en la segregación de la hormona natural del crecimiento llamado somatotropina la cual actúa directamente sobre las células estimulando el crecimiento y favoreciendo el metabolismo proteico. Este favorece un mayor incremento de las masas musculares dando un mayor aumento de peso y una mejor eficiencia alimenticia. Asimismo el sistema óseo crecerá a su máximo tamaño genético.

La Comercial Solverst Corporation (1971) afirma que el Ralgro produce aumento de peso diario hasta en un $18 \%$ y reduce la cantidad de alimento requerido por cada $\mathrm{kg}$ de aumento de peso en un $10.2 \%$. Igualmente Hoechst Peruana (1985) encontró, en pruebas de alimentación en ovinos, aumentos de 15 a $30 \%$ con relación al testigo. También coincidió con estos resultados A macifuen (1991) trabajando con ovinos de la raza Pelibuey, en Tingo María. Este investigador encontró que al aplicar el zeranol la ganancia de peso fue mayor en un $19 \%$ respecto al testigo. También se obtuvieron mejores rendimientos de carcasa y mayores beneficios económicos netos.

Por otro lado, en trabajos realizados en la sierra con ovinos con manejo intensivo y con pastos cultivados, Castillo (1986) encontró que el implante de zeranol fue superior en un $26 \%$ respecto al testigo, siendo la ganancia de peso ( $\mathrm{g}$. animal ${ }^{-1} \mathrm{di}^{-1}$ ) de 165.7 y 145.0 para los tratamientos con implante y testigo, respectivamente.

\section{MATERIALES Y METODOS}

\section{LOCALIZACION}

El estudio se realizó durante 63 días entre julio y setiembre de 1991, en la Estación Experimental "Villa Rica" del Centro Regional de Investigación del 
Instituto de Investigaciones de la A mazonía Peruana, a $12.4 \mathrm{~km}$ de la ciudad de Pucallpa. La Estación está geográficamente ubicada a 80 22'23' y 74은 34' y a una altitud de $154 \mathrm{msnm}$. La precipitación media anual es de $1852 \mathrm{~mm}$., la temperatura promedio de $25.5 \circ \mathrm{C}$ y la humedad relativa de $82 \%$.

\section{ANIMALES}

Se usaron 16 ovinos machos de pelo de la raza Pelibuey con una edad de tres meses y con un peso promedio de $15.21 \mathrm{~kg}$. Los 16 ovinos fueron distribuidos al azar en cuatro grupos de cuatro animales cada uno.

\section{INSTALACIONES}

Se utilizó un aprisco de $32 \mathrm{~m} 2$ construido con techo de techalit, piso y cerco enrejillado de madera dividido en cuatro corrales de ocho m2 cada uno. Cada corral fue equipado con bebedero y comedero de pasto y alimento balanceado, respectivamente.

\section{TRATAMIE NTOS}

Cada grupo de animales fueron alimentados con cuatro raciones diferentes

$\mathrm{T} 1$ = Pastos solo (Testigo)

$\mathrm{T} 2$ = Pasto + Alimento Balanceado

T3 = Pasto + Alimento Balanceado + Zeranol

14 = Pasto + Zeranol

\section{ALIMENTACION Y MANEJ 0}

Todos los animales fueron pastoreados diariamente desde las 07:00 hasta las 13:00 horas en una pastura de Brachiaria decumbens, luego se les suministro $2 \mathrm{Kg}$. de pasto $\mathrm{B}$. decumbens cortado por animal cada día a los tratamientos Ti y $14,500 \mathrm{~g}$ por animal cada día de alimento balanceado con $16.49 \%$ proteína total para los tratamientos T2 y T3. El agua fue suministrada ad libiturn. La implantación de

zeranol fue hecha vía subcutánea con $12 \mathrm{mg}$ del producto por cada animal.

Los animales fueron pesados semanalmente en ayunas durante todo el experimento, finalmente fueron sacrificados para las evaluaciones del rendimiento de carcasa. 


\section{VARIABLES MEDIDAS}

Fueron ganancia de peso, rendimiento de carcasa y beneficio económico.

\section{DISE ÑO E STADÍSTICO}

Se usó el diseño completamente al azar con cuatro repeticiones.

\section{RESULTADOS}

\section{GANANCIA DE PESO}

El efecto del zeranol sobre los pesos corporales promedios, así como sobre la ganancia de peso de los ovinos se muestra en el cuadro 1 . Se encontró que el T3

(Pasto + Alimento Balanceado + Zeranol) fue superior a los demás tratamientos $(P<0.05)$. No existieron diferencias significativas entre el tratamiento T2 (Pasto $+$

Alimento Balanceado) y el T4 (Pasto + Zeranol), pero ambas fueron superiores al

T1 (Pasto solo).

\section{Cuadro 1}

Peso inicial, final y ganancia diaria de peso de los ovinos Pelibuey ${ }^{1}$ en engorde en un período de 63 días. Promedio de cuatro animales

\begin{tabular}{|c|c|c|c|}
\hline \multirow[b]{2}{*}{ Tratamiento } & \multicolumn{2}{|c|}{ Peso } & \multirow{2}{*}{$\begin{array}{l}\text { Ganancia } \\
\text { diaria }\end{array}$} \\
\hline & Inicial & Final & \\
\hline & \multicolumn{2}{|c|}{ 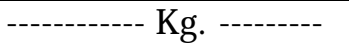 } & - \\
\hline Pasto solo & 15.64 & 23.25 & $0.121 \mathrm{e}^{3}$ \\
\hline Pasto + AB2 & 15.00 & 24.35 & $0.149 b$ \\
\hline Pasto + AB + Zeranol & 15.00 & 26.38 & $0.181 a$ \\
\hline Pasto + Zeranol & 15.19 & 24.48 & $0.148 b$ \\
\hline
\end{tabular}

1. Ovinos de 3 meses de edad en promedio, al inicio del estudio

2. A limento balanceado

3. V alores seguidos por letras iguales no difieren significativamente $(P<0.05)$ 


\section{RENDIMIENTO DE CARCASA}

El rendimiento de carcasa de los ovinos Pelibuey, según los tratamientos en estudio se muestra en el cuadro 2. Se encontraron diferencias significativas siendo superior $(P=0.05)$ el T3. No se encontraron diferencias significativas entre los tratamientos T2 y T4, pero ambos fueron superiores al tratamiento T1.

\section{Cuadro 2.}

Efecto del zeranol sobre el rendimiento de carcasa de los ovinos Pelibuey en engorde, por un período de 63 días. Promedio de cuatro animales.

\begin{tabular}{lc}
\hline Tratamiento & $\begin{array}{c}\text { Rendimiento de } \\
\text { Carcasa }\end{array}$ \\
\hline Pasto solo & $45.75 \mathrm{c}^{1}$ \\
Pasto + Alimento Balanceado & $48.25 \mathrm{~b}$ \\
Pasto + Alimento Balanceado + Zeranol & $50.47 \mathrm{a}$ \\
Pasto + Zeranol & $48.21 \mathrm{~b}$ \\
\hline
\end{tabular}

$1 \mathrm{~V}$ alores seguidos por letras iguales no difieren significativamente $(P<0.05)$

\section{ANÁLISIS ECONOMICO}

Para la evaluación económica del experimento se determinó el beneficio neto por animal, mediante la siguiente fórmula

donde:

$$
B=P Y-(C V+C F)
$$

$$
\begin{aligned}
& B=\text { Beneficio neto en soles por ovino } \\
& P=\text { Precio por kg de ovino en soles } \\
& Y=\text { Peso promedio por ovino por tratamiento } \\
& C V=\quad \text { Costos variables por ovino por tratamiento } \\
& C F=\quad \text { Costos fijos por ovino por tratamiento }
\end{aligned}
$$

Los resultados se presenta en el cuadro 3 donde se observa que el tratamiento más rentable fue el tratamiento T3, seguido por los tratamientos T2 T4 y T 1, respectivamente. 


\section{C uadro 3}

A nálisis económico del engorde ovinos Pelibuey en un período de 63 días usando zeranol.

\begin{tabular}{lllll}
\hline Rubro & TI & $\begin{array}{l}\text { Tratamiento } \\
\text { T2 }\end{array}$ & T3 & T4 \\
\hline $\begin{array}{l}\text { Precio por kg } \\
\text { de ovino (S/.) }\end{array}$ & 1.20 & 1.20 & 1.20 & \\
1.20 & & & & \\
$\begin{array}{l}\text { Peso promedio por } \\
\text { ovino (kg) }\end{array}$ & 23,250 & 24,350 & 26,375 & 24,475
\end{tabular}

Ingreso Bruto por

$\begin{array}{llll}\text { ovino }(\mathrm{S} / .) & 27.90 & 29.22 & 31.65\end{array}$

29.85

Costo variable por ovino(S/.)

10.82

10.08

9.45

10.19

Costo Fijo

por ovino (S/.)

9.23

9.23

9.23

9.23

Beneficio N eto:

$\begin{array}{llll}\text { Por animal } & 8.59 & 10.54 & 12.23\end{array}$

9.80

Por kg de PV de

carne

0.369

0.432

0.463

0.380

* Este costo se realizó al cambio de S/. 0.790 de dólar 


\section{DISCUSION}

\section{GANANCIA DE PESO}

La ganancia de peso de T3 fue superior en $17.8 \%$ al T2, en un $18.4 \%$ al $\mathrm{T} 4$, yen un $33.1 \%$ al T1. Menores ganancias de peso pero con igual tendencia fueron encontradas por A macifuen (1991) quien obtuvo ganancias de peso diario de 91; 68; y 55 g usando Pasto + Zeranol + Sales M inerales, Pasto + Zeranol y Pasto solo, respectivamente, en ovinos Pelibuey. Laza (1985) reporté ganancias de peso diario de $113.08 \mathrm{~g}$ en ovinos de lana al usar sal común e implante de zeranol. La mayor ganancia de peso del tratamiento T3 se atribuye al efecto combinado del alimento balanceado más el zeranol, que al actual sobre la glándula pituitaria estimula la hormona de crecimiento para una mayor síntesis proteica. La ausencia de diferencias significativas entre el tratamiento T2 y el T4 se debería a que en el T4 el anabolizante favoreció la mayor retención y asimilación de nutrientes contenidos en el pasto y en el T2, los animales aparte del consumo del pasto, cubrieron sus requerimientos nutricionales con el alimento balanceado.

\section{RENDIMIENTO DE CARCASA}

El mayor rendimiento de carcasa del tratamiento T3 fue debido posiblemente a que el zeranol estimuló la mayor retención de nitrógeno contenido en el pasto y el alimento balanceado, lo cual incrementé las masas musculares y el tejido óseo (Castillo, 1986), dando como resultado un mayor rendimiento de carcasa a los animales implantados. Estos rendimientos son superiores a 10 encontrado por Combellas (1980), cuyo rendimiento de carcasa fue de $44.9 \%$ en ovinos Pelibuey.

\section{ANALISIS ECONOMICO}

El tratamiento T3 (Pasto solo + Alimento Balanceado + Zeranol) fue el de mayor rendimiento económico. Esto fue mayor en un $6.7 ; 17.9$ y $20.3 \%$ que los tratamientos $\mathrm{T} 2, \mathrm{~T} 4$ y $\mathrm{T} 1$, respectivamente. Esta ventaja económica se debería al efecto aditivo del zeranol, que permitió un mejor aprovechamiento del pasto y alimento balanceado, Io que se reflejó en el mayor incremento de peso y por consiguiente mayor ingreso total neto. 


\section{CONCLUSIONES}

Con base en los resultados de este estudio se puede concluir que la mejor ganancia de peso, rendimiento de carcasa y beneficio económico se obtuvo al usar implante zeranol en el engorde de ovinos Pelibuey.

\section{BIBLIOGRAFIA}

AMACIFUEN, C. 1991. Evaluación del efecto biológico y económico del anabólico zeranol en el engorde de ovinos de pelo en sistema de crianza intensiva en Tingo María. Tesis Ing. Zootecnista, Universidad $\mathrm{Nacional}$ A graria de la Selva, Tingo M aría.

CASTILLO, F.J. 1986. Estudio comparativo del engorde de capones vs caponcillos en pastos cultivados con implante de zeranol en la Cooperativa Comunal Sacra Familia. Tesis Ing. Zootecnista, Universidad Nacional Daniel Alcides Carrión, Cerro de Pasco, Perú.

COMERCIAL SOLVERST CORPORATION. 1971. Implante Ralgro para ganado vacuno y ovino en el Perú. Boletín Informativo.

COMBELLAS DE J. 1980. Parámetros productivos y reproductivos de ovejas tropicales en sistemas de pasturas mejoradas. Revista Producción A nimal Tropical, V enezuela, 5(3):29 1-297.

HOECHST PERUANA S.A. 1985. Ralgro. Boletín Informativo. Lima. Perú,

INTERNATIONAL MINERAL CHEMICAL CORPORATION 1982. Respuestas a sus preguntas sobre implante de Ralgro. Torre Hause. $1 \mathrm{p}$.

LAO,A.L. 1985. Engorde de borregas de saca en una mezcla de pastos cultivados en Sacra Familia. Tesis. Ing. Zooetecnista, Universidad Nacional Daniel Alcides Carrión, Cerro de Pasco, Perú.

VARA, 0.; MORENO, R. 1988. Sistema de engorde vacuno. 2da. ed. U niversidad Nacional A graria, La M olina. Lima, Perú. 124 p. 